\title{
Electrically Benign Defect Behavior in Zinc Tin Nitride Revealed from First Principles
}

\author{
Naoki Tsunoda, ${ }^{1}$ Yu Kumagai, ${ }^{2,3,}{ }^{*}$ Akira Takahashi, ${ }^{1}$ and Fumiyasu Oba ${ }^{1,2,4}$ \\ ${ }^{1}$ Laboratory for Materials and Structures, Institute of Innovative Research, Tokyo Institute of Technology, \\ Yokohama 226-8503, Japan \\ ${ }^{2}$ Materials Research Center for Element Strategy, Tokyo Institute of Technology, Yokohama 226-8503, Japan \\ ${ }^{3}$ PRESTO, Japan Science and Technology Agency, Tokyo 113-8656, Japan \\ ${ }^{4}$ Center for Materials Research by Information Integration, Research and Services Division of Materials Data and \\ Integrated System, National Institute for Materials Science, Tsukuba 305-0047, Japan
}

(Received 19 April 2018; revised manuscript received 1 June 2018; published 2 July 2018)

\begin{abstract}
Zinc tin nitride $\left(\mathrm{ZnSnN}_{2}\right)$ is attracting growing interest as a nontoxic and earth-abundant photoabsorber for thin-film photovoltaics. Carrier transport in $\mathrm{ZnSnN}_{2}$ and consequently cell performance are strongly affected by point defects with deep levels acting as carrier recombination centers. In this study, the point defects in $\mathrm{ZnSnN}_{2}$ are revisited by careful first-principles modeling based on recent experimental and theoretical results. It is shown that $\mathrm{ZnSnN}_{2}$ does not have low-energy defects with deep levels, in contrast to previously reported results. Therefore, $\mathrm{ZnSnN}_{2}$ is more promising as a photoabsorber material than formerly considered.
\end{abstract}

DOI: 10.1103/PhysRevApplied.10.011001

$\mathrm{ZnSnN}_{2}$ has a wurtzite-derived structure with a minimum direct band gap of $1.4 \mathrm{eV}[1,2]$, which is very close to the ideal Shockley-Queisser value of $1.34 \mathrm{eV}$ [3]. In addition, the electron effective mass of $0.17 m_{0}$ and its heavyhole mass of $2.00 m_{0}$ are comparable or even superior to those of GaN (Fig. S2, Supplemental Material [4], and [5]). However, there are several fundamentally and technologically important issues regarding the use of $\mathrm{ZnSnN}_{2}$ as a light absorption layer that need to be assessed: (i) Point-defect properties. It is necessary to identify any point defects with deep levels, because they trap electrons and/or holes and cause carrier recombination, leading to loss of cell efficiency. (ii) Unintentional n-type doping. The carrier electron concentration generally unintentionally increases up to $10^{21} \mathrm{~cm}^{-3}$ in $\mathrm{ZnSnN}_{2}$ as well as other narrow-gap nitrides like $\mathrm{InN}, \mathrm{Zn}_{3} \mathrm{~N}_{2}$, and $\mathrm{ScN}$ [6-8]. For solar-cell applications, the carrier electron concentration needs to be lowered to around $10^{16}-10^{18} \mathrm{~cm}^{-3}$. Besides, the photoabsorption onset is increased up to $2.4 \mathrm{eV}[1,9,10]$ because of the Burstein-Moss (BM) shift [11,12], which raises considerable controversy regarding the fundamental gap

*yuuukuma@gmail.com

Published by the American Physical Society under the terms of the Creative Commons Attribution 4.0 International license. Further distribution of this work must maintain attribution to the author(s) and the published article's title, journal citation, and DOI. of $\mathrm{ZnSnN}_{2}$. The BM shift is also directly related to the formation of donor-type point defects. Understanding the point-defect properties of $\mathrm{ZnSnN}_{2}$ is thus imperative to optimize its performance in photovoltaics.

Chen et al. [13] investigated native point defects and oxygen impurities in $\mathrm{ZnSnN}_{2}$ with an ordered orthorhombic structure. They report that $\mathrm{Sn}$-on-Zn antisite $\left(\mathrm{Sn}_{\mathrm{Zn}}\right)$ and $\mathrm{O}$-on-N $\left(\mathrm{O}_{\mathrm{N}}\right)$ substitutional defects have low formation energies with deep donor levels and thus do not cause the $\mathrm{BM}$ shift in $\mathrm{ZnSnN}_{2}$. However, given recent experimental and theoretical findings $[1,2,9,10,14-16]$, this conclusion needs to be reviewed. This motivated us to reevaluate point defects in $\mathrm{ZnSnN}_{2}$ including currently unexplored hydrogen impurities. Our results show that all the defects with deep levels are very high in energy and the abundant defects act as shallow donors. Considering these favorable defect properties together with its appropriate band gap and high absorption coefficient, $\mathrm{ZnSnN}_{2}$ is a promising photoabsorber for thin-film photovoltaics.

Our theoretical investigation of the point defects in $\mathrm{ZnSnN}_{2}$ is performed for the $P n a 2_{1}$ orthorhombic structure with 16 atoms in the unit cell, referred to here as the ordered model. However, a certain level of disorder in the cation sublattice appears to be unavoidable because of the very low cation order-disorder transition temperature [2]. At low temperature, the disordered phase strictly retains the local charge neutrality, in which each $\mathrm{N}$ atom is necessarily coordinated by two Sn and two $\mathrm{Zn}$ atoms. We refer to such a structure as the disordered structure 
with local charge neutrality (DLCN). It is reported that the electronic structure of the DLCN is almost identical to that of the ordered structure $[2,15]$. Thus, the DLCN should be ideal as a photoabsorber, unless its defects are detrimental to its efficiency. We thus consider both ordered and DLCN models in our defect calculations. Note that cation disorder breaks local charge neutrality at very high temperature $(>1750 \mathrm{~K}$ [2]). Some authors use special quasirandom structures (SQS) to examine the behavior of the cation disordered phase $[9,10]$. Such a fully random cation disordered model is not appropriate for $\mathrm{ZnSnN}_{2}$ because its typical growth temperature is much lower than $1750 \mathrm{~K}$ $[1,9,10,14,16]$. Besides, when the charge neutrality is broken, a high concentration of $\mathrm{Zn}$-on-Sn $\left(\mathrm{Zn}_{\mathrm{Sn}}\right)$ defects is introduced. This situation should be avoided for photovoltaic applications because $\mathrm{Zn}_{\mathrm{Sn}}$ defects give rise to deep levels, as discussed later.

Figure 1 shows the two models used to evaluate defect formation energies. The DLCN model is generated by Monte Carlo-simulated annealing of a 128-atom orthorhombic supercell. The DLCN model shown in Fig. 1 belongs to space group $\mathrm{Pna}_{1}$ and consists of a 32-atom unit cell, consistent with that reported elsewhere [2]. Note that there are infinite possible configurations for the DLCN models when different cell sizes are considered; however, within the 128-atom supercell, we found only one configuration owing to the strong geometrical constraint of the local charge neutrality.

First, we discuss the band gap of $\mathrm{ZnSnN}_{2}$ because it is a central controversy in the research of this material.
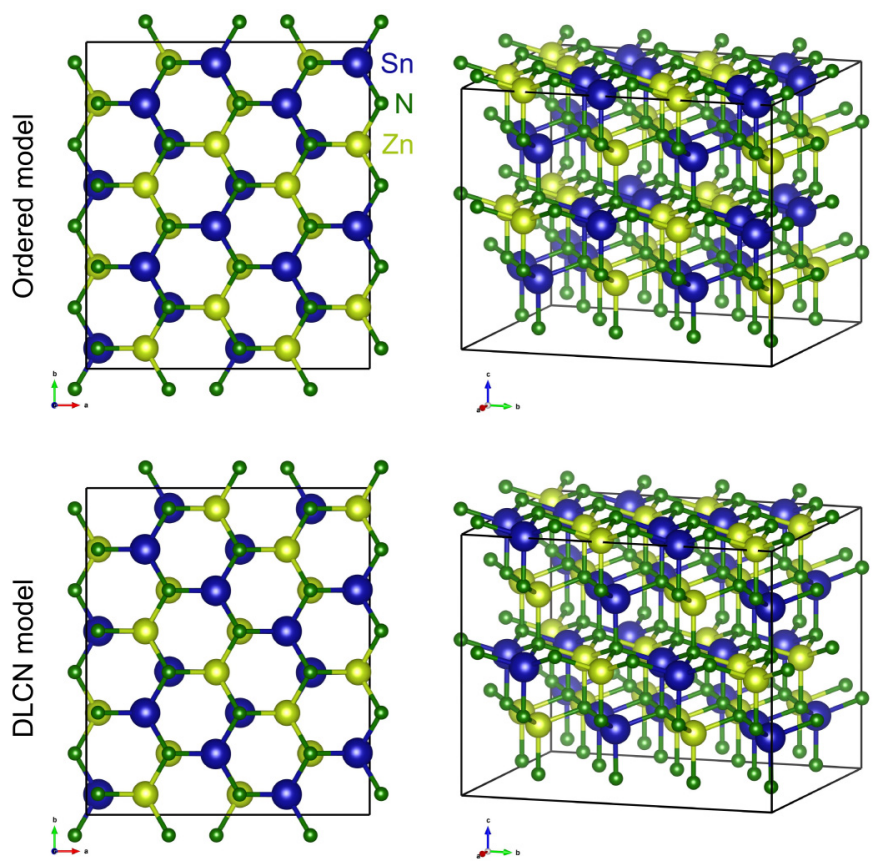

FIG. 1. Atomic structures of the ordered and DLCN models of $\mathrm{ZnSnN}_{2}$ used in this study. These models contain 128 atoms. Both structures belong to space group $P n a 2_{1}$.
As the carrier electron concentration increases, the photoabsorption onset also increases due to the BM shift. The blueshift estimated from the Heyd-Scuseria-Ernzerhof (HSE06) hybrid functional calculations [31,32] is 0.2-2.3 $\mathrm{eV}$ when the carrier electron concentration is $10^{18}-10^{21}$ $\mathrm{cm}^{-3}$ observed in experiments $[1,9,10]$ (Fig. S6, Supplemental Material [4]). Thus, larger experimental band gaps than $1.4 \mathrm{eV}$ could be attributed to the BM shift, as is also discussed by Lahourcade et al. [1] Second, the band gaps of fully cation-disordered systems are ill-defined because the minimum gap decreases as the model size increases [17]. In the case of $\mathrm{ZnSnN}_{2}$, the density of states tends to continuously develop within the band gap when $\mathrm{N}$ atoms are coordinated by three or four $\mathrm{Sn}$ or $\mathrm{Zn}$ atoms, as discussed later. Note that this does not necessarily indicate metallic behavior because the defect states are discontinuous in real space. Refer to the Supplemental Material for a more detailed discussion considering SQS (Fig. S8 [4]).

We now discuss the energetics of point defects in $\mathrm{ZnSnN}_{2}$. To investigate the site dependency in the DLCN model, we conduct over 300 point-defect calculations using the modified Perdew-Burke-Ernzerhof generalized gradient approximation tuned for solids (PBEsol GGA) [18]. We confirm that most of the defect species show only negligible site dependencies in energy, while the interstitial defects exhibit relatively large ones, probably due to the difference in neighboring cation configurations at the octahedral sites (Fig. S9, Supplemental Material [4]). Thus, we perform HSE06 calculations for a few configurations with lower energies in PBEsol calculations for each defect and show only the results of the lowest-energy configurations hereafter. Figure 2 shows the resultant formation energies of the native defects as well as hydrogen and oxygen impurities, which are ubiquitous in nitrides. Since $\mathrm{ZnSnN}_{2}$ is only slightly more stable $\left(\Delta H_{f}=-0.015 \mathrm{eV} /\right.$ atom $)$ than $\mathrm{Zn}$ metal, Sn metal, and $\mathrm{N}_{2}$ molecules, based on HSE06 calculations, the growth condition does not significantly affect the formation energies under the equilibrium condition. Therefore, here, the chemical potentials of constituent elements are set at the point where $\mathrm{Zn}$ and $\mathrm{Sn}$ metals equilibrate with $\mathrm{ZnSnN}_{2}$. As mentioned above, the standard HSE06 functional used in this study yields a direct gap of $1.4 \mathrm{eV}$. In the study by Chen et al., the Fock exchange ratio is increased to reproduce a reported experimental gap of $2.0 \mathrm{eV}$ [1], although one of the authors attributes this larger gap to the BM shift and states that the fundamental gap is about $1.4 \mathrm{eV}$ [1].

Before discussing the results, let us consider the technical details that can alter conclusions even qualitatively. Chen et al. [13] did not consider the finite cell-size effects when calculating defect formation energies and the reciprocal-space sampling is performed using only the $\Gamma$ point. According to our calculated cellsize dependencies, a maximum error of $0.72 \mathrm{eV}$ arises when the 128-atom supercell is used with these settings. 

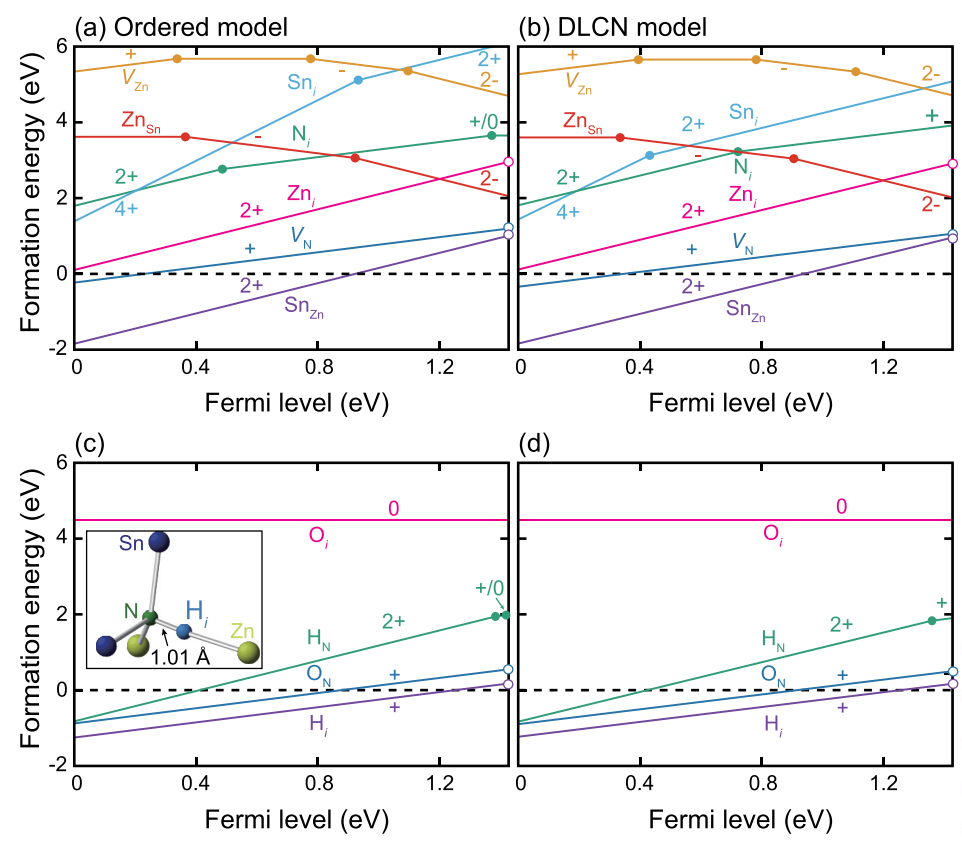

(d)

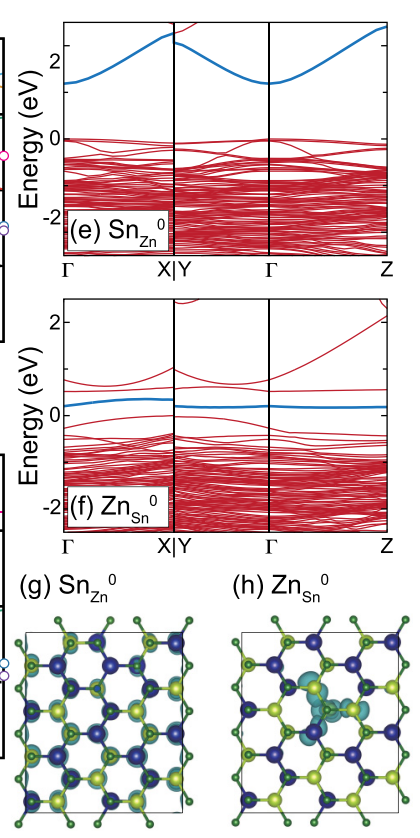

FIG. 2. Formation energies of point defects in $\mathrm{ZnSnN}_{2}$ as a function of the Fermi level. (a),(b) Native defects and (c),(d) impurities in the (a),(c) ordered model and (b),(d) DLCN model. The valence-band maximum is set to the zero of the Fermi level and the upper limit corresponds to the conduction-band minimum. The defect species and sites are indicated by $X_{Y}$, where $X$ means a vacancy $(V)$ or an added element and $Y$ is an interstitial site $(i)$ or substitutional site. The chemical potentials are set at a point where the $\mathrm{Zn}$ and $\mathrm{Sn}$ metals equilibrate with $\mathrm{ZnSnN}_{2}$. Note that shallow donor levels in the vicinity of the conduction-band minimum are designated by open circles (see text in detail). Note also that the Sn vacancy does not appear in panels (a) and (b) owing to its very high formation energy (see Fig. S12, Supplemental Material). The inset of (c) shows the atomic structure for $\mathrm{H}_{i}^{+}$. (e),(f) Band structures for the $\mathrm{Sn}_{\mathrm{Zn}}^{0}$ and $\mathrm{Zn}_{\mathrm{Sn}}^{0}$ in the 128-atom supercell of the ordered model. Note that $\mathrm{Zn}_{\mathrm{Sn}}^{0}$ shows spin polarization, and only the down-spin channel, which indicates two unoccupied deep localized states, is shown (see Fig. S14, Supplemental Material, for the up-spin channel). (g),(h) Squared wave functions of the highest occupied and the lowest unoccupied bands for $\mathrm{Sn}_{\mathrm{Zn}}^{0}$ and $\mathrm{Zn}_{\mathrm{Sn}}^{0}$, respectively, highlighted by blue color in panels (e) and (f).

In this study, we decrease these errors using the extended Freysoldt-Neugebauer-Van de Walle (FNV) corrections $[19,20]$ and $2 \times 2 \times 2$ Monkhorst-Pack $k$-point sampling for the 128-atom supercell. Consequently, the errors based on our test calculations are less than $0.06 \mathrm{eV}$. In the case of defects exhibiting hydrogenic states, a huge supercell including tens of thousands of atoms is usually required to avoid overlap between widespread defect orbitals [21]. When using the 128-atom supercell in $\mathrm{ZnSnN}_{2}$, the thermodynamic transition levels of such defects are overestimated by a few tenths of an $\mathrm{eV}$ because of the defect-defect interaction (see Fig. S10, Supplemental Material [4]). Therefore, we discuss the transition levels associated with hydrogenic states only qualitatively.

Figures 2(a)-2(d) reveal that there is little difference between the defect formation energies of the ordered and DLCN models, which means that not only the bulk properties but also the point-defect properties are strongly correlated with its immediate coordination environment. This conclusion would also hold for similar cation-disordered systems such as $\mathrm{ZnSnP}_{2}$ [22,23] and $\mathrm{ZnGeN}_{2}$ [24] if the local charge neutrality is preserved. It is also notable that defects and impurities that show low formation energies simultaneously with deep levels do not exist when the Fermi level is located within the band gap. This is in stark contrast to the conclusion of a previous study [13], that the $\mathrm{Zn}$ interstitial $\left(\mathrm{Zn}_{i}\right), \mathrm{Sn}_{\mathrm{Zn}}$, nitrogen vacancy $\left(V_{\mathrm{N}}\right)$, and $\mathrm{O}_{\mathrm{N}}$ have deep defect levels and act as carrier recombination centers. This discrepancy is mainly caused by the aforementioned difference in the treatment of the cell-size corrections and $k$-point sampling.

As shown in Figs. 2(e) and 2(g), $\mathrm{Sn}_{\mathrm{Zn}}^{0}$ exhibits an occupied hydrogenic state, namely perturbed host state, while the localized defect state is not confirmed near the conduction-band minimum (CBM), which is different from the results by Chen et al. [13] Therefore, $\mathrm{Sn}_{\mathrm{Zn}}$ is a dominant shallow donor among the native defects. Its formation energy becomes zero at the Fermi level that is $0.9 \mathrm{eV}$ above the valence-band maximum (VBM) and hinders $p$-type conversion even with acceptor doping. $V_{\mathrm{N}}$ is a single shallow donor in the entire Fermi-level range but has a higher formation energy than that of $\mathrm{Sn}_{\mathrm{Zn}}$. In contrast, acceptor-type defect $\mathrm{Zn}_{\mathrm{Sn}}$ forms two deep transition levels between the $0,-1$, and -2 charge states. 
Indeed, as seen in Figs. 2(f) and 2(h), $\mathrm{Zn}_{\mathrm{Sn}}^{0}$ shows two deep localized defect states within the band gap. However, the formation energy of $\mathrm{Zn}_{\mathrm{Sn}}$ is rather high when the Fermi level is within the band gap. The other defects, namely, $\mathrm{Zn}$ vacancy $\left(V_{\mathrm{Zn}}\right)$, Sn vacancy $\left(V_{\mathrm{Sn}}\right)$, Sn interstitial $\left(\mathrm{Sn}_{i}\right)$, and $\mathrm{N}$ interstitial $\left(\mathrm{N}_{i}\right)$, create deep levels but have very high formation energies. It is also noteworthy that $\mathrm{Sn}_{\mathrm{Zn}}^{0}$ does not become a $D X$ center $\left(V_{\mathrm{Zn}}+\mathrm{Sn}_{i}\right)$ in our calculations unlike $\mathrm{ZnSnP}_{2}$ [22], probably because of the high formation energies of $V_{\mathrm{Zn}}$ and $\mathrm{Sn}_{i}$ and/or the difference in crystal structures.

Oxygen and hydrogen impurities are energetically favorable at the $\mathrm{N}$ sites $\left(\mathrm{O}_{\mathrm{N}}\right)$ and interstitial sites $\left(\mathrm{H}_{i}\right)$, respectively. These impurities also act as single shallow donors and exhibit low formation energies even when the Fermi level is at the CBM. Orbital analyses indicate that their donor electrons are located at the perturbed conduction bands. Therefore, these impurities should primarily cause the BM shift. However, in the previous study [13], the origin of the high carrier electron concentration is attributed to the defect band originating from $\mathrm{Sn}_{\mathrm{Zn}}$ and $\mathrm{O}_{\mathrm{N}}$, which is different from our results. $\mathrm{H}_{i}$ forms an $\mathrm{N}-\mathrm{H}$ bond (1.01 $\AA$ ) between the $\mathrm{N}$ and $\mathrm{Zn}$ atoms, as commonly observed for nitrides. We also find that anionic hydrogen $\mathrm{H}_{i}^{-1}$ is not stabilized even when the Fermi level is located at $1 \mathrm{eV}$ above the CBM in $\mathrm{ZnSnN}_{2}$ (see Fig. S12, Supplemental Material [4]), unlike in GaN [25].

In a recent paper, Fioretti et al. [16] show that annealing $\mathrm{Zn}$-rich $\mathrm{Zn}_{1+x} \mathrm{Sn}_{1-x} \mathrm{~N}_{2}$ grown in a hydrogen atmosphere decreases its carrier electron concentration to $4 \times$ $10^{16} \mathrm{~cm}^{-3}$. They explain this observation from the viewpoint of hydrogen passivation of acceptors, i.e., $\mathrm{Zn}_{\mathrm{Sn}}+\mathrm{H}_{i}$ in $\mathrm{Zn}_{1+x} \mathrm{Sn}_{1-x} \mathrm{~N}_{2}$ during growth, which lowers the driving force for the formation of other unintentional donors. Indeed, our results indicate that complexing with hydrogen is exothermic and drastically decreases the formation energy of acceptor $\mathrm{Zn}_{\mathrm{Sn}}$ (Fig. S13 [4]); the binding energy, i.e., the energy change from isolated $\mathrm{Zn}_{\mathrm{Sn}}^{-2}$ and $\mathrm{H}_{i}^{+}$to $\left(\mathrm{Zn}_{\mathrm{Sn}}+\mathrm{H}_{i}\right)^{-}$is $-1.43 \mathrm{eV}$ and that from isolated $\mathrm{H}_{i}^{+}$and $\left(\mathrm{Zn}_{\mathrm{Sn}}+\mathrm{H}_{i}\right)^{-}$to $\left(\mathrm{Zn}_{\mathrm{Sn}}+2 \mathrm{H}_{i}\right)^{0}$ is $-0.73 \mathrm{eV}$. Therefore, abundant $\mathrm{Zn}_{\mathrm{Sn}}$ antisites are easily introduced by hydrogen passivation as Fioretti et al. discuss. We, however, emphasize that $\mathrm{Zn}_{\mathrm{Sn}}$ antisites, which generate deep transition levels and trap minority carrier holes, persist even after removing the passivating hydrogen by postdeposition annealing. Moreover, the determination of the intrinsic band gap would be inhibited by the optical absorption related to the defect band.

Here, we propose an alternative route to achieving $\mathrm{ZnSnN}_{2}$ with lower carrier electron concentration. In the above discussion, the chemical potentials are set at a point where the $\mathrm{Zn}$ and $\mathrm{Sn}$ metals equilibrate with $\mathrm{ZnSnN}_{2}$. However, recent growth techniques such as reactive sputtering can be used to raise the chemical potential of $\mathrm{N}\left(\mu_{\mathrm{N}}\right)$ by up to $+1 \mathrm{eV} / \mathrm{N}$ from that of an inactive
$\mathrm{N}_{2}$ molecule [26]. Consequently, metastable nitrides can be synthesized. A notable example is $\mathrm{Cu}_{3} \mathrm{~N}$, in which $\mu_{\mathrm{N}}$ needs to be at least $+1.04 \mathrm{eV}$ higher than that of an $\mathrm{N}_{2}$ molecule [26]. When $\mu_{\mathrm{N}}$ is increased to this value, we can use more advantageous conditions for lowering the carrier electron concentration. Figure 3 shows the formation energies of native defects and oxygen impurity under $\Delta \mu_{\mathrm{N}}=$ $+1 \mathrm{eV} / \mathrm{N}$ and Zn-rich (Sn-poor) conditions (see Fig. S16 for details [4]). The equilibrium Fermi level is located at $0.70 \mathrm{eV}$ from the $\mathrm{CBM}$ at $600 \mathrm{~K}$, which assumes synthesis temperature of $\mathrm{ZnSnN}_{2}$ [9,14]. In this situation, all the defect concentrations are less than $10^{14} \mathrm{~cm}^{-3}$ and the corresponding carrier concentration is $n=1.9 \times 10^{14} \mathrm{~cm}^{-3}$. One might expect that $p$-type conversion by acceptor doping is plausible in this condition as all the donor-type defects compensating holes are high in energy. However, it is found from extensive calculations for the impurities $\mathrm{Li}, \mathrm{Na}, \mathrm{K}, \mathrm{Cu}$, and $\mathrm{C}$ using the HSE06 functional (see Fig. S17, Supplemental Material [4]) that none of them act as effective acceptor dopants for $\mathrm{ZnSnN}_{2}$ because of deep acceptor levels or incorporation into interstitial sites. Thus, $p$-type conversion in $\mathrm{ZnSnN}_{2}$ seems very difficult, as previously considered, even if compensating donor-type defects are sufficiently suppressed as in Fig. 3 [27].

Methods. First-principles calculations are performed using the projector augmented-wave (PAW) method $[28,29]$ as implemented in VASP [30]. The PBEsol GGA [18] is used to calculate the total energies of various DLCN models and SQS with different sizes, and to check the site and cell-size dependencies of defect formation energies. The HSE06 hybrid functional $[31,32]$ with standard parameters is used for the calculations of the band structure, density of states, and defect formation energies. The plane-wave cutoff energy is set to $550 \mathrm{eV}$ for the lattice relaxation of the ordered and DLCN models without

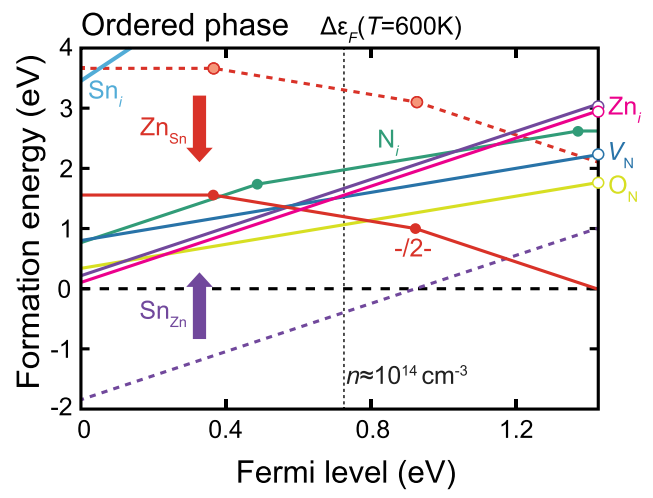

FIG. 3. Same as Fig. 2, but the nitrogen chemical potential is increased by $1 \mathrm{eV}$ under the Zn-rich condition (see text for details). Since hydrogen impurity could be reduced by postgrowth annealing, we consider only oxygen as an impurity, here. For comparison, the dashed lines show the $\mathrm{Sn}_{\mathrm{Zn}}$ and $\mathrm{Zn}_{\text {Sn }}$ formation energies under the $\mathrm{Zn}$-rich and $\mathrm{Sn}$-rich condition considered in Fig. 2. 
defects and $400 \mathrm{eV}$ for the other calculations with fixed lattice constants. The residual forces are converged to less than 0.01 and $0.04 \mathrm{eV} / \AA$ for the calculations without and with defects, respectively. Spin polarization is considered for all the defect calculations using the HSE06 functional. [19]

The formation energy of a point defect is calculated as

$$
\begin{aligned}
E_{f}\left[D^{q}\right]= & \left\{E\left[D^{q}\right]+E_{\mathrm{corr}}\left[D^{q}\right]\right\}-E_{P}+\sum n_{i} \mu_{i} \\
& +q\left(\epsilon_{\mathrm{VBM}}+\Delta \epsilon_{F}\right),
\end{aligned}
$$

where $E\left[D^{q}\right]$ and $E_{P}$ are the total energies of the supercell with defect $D$ in charge state $q$ and the supercell without a defect, respectively. $n_{i}$ is the number of removed $\left(n_{i}>0\right)$ or added $\left(n_{i}<0\right) i$-type atoms and $\mu_{i}$ is the chemical potential representing the growth conditions. The referenced competing phases used are hexagonal $\mathrm{Zn}$, cubic $\mathrm{Sn}$, wurtzite $\mathrm{ZnO}$, and $\mathrm{N}_{2}$ and $\mathrm{H}_{2}$ molecules. $\epsilon_{\mathrm{VBM}}$ is the energy level of the VBM, and $\Delta \epsilon_{F}$ is the Fermi level $\left(\epsilon_{F}\right)$ with respect to $\epsilon_{\mathrm{VBM}}$. Therefore, $\epsilon_{F}=\epsilon_{\mathrm{VBM}}+\Delta \epsilon_{F}$. $E_{\text {corr }}\left[D^{q}\right]$ corresponds to the correction energy for a finite supercell-size error associated with spurious electrostatic interactions between charged defects. We use our extended FNV correction scheme [19] in the calculations. More computational details are described in the Supplemental Material [4].

Both DLCN and SQS models are generated by Monte Carlo-simulated annealing using in-house and CLUPAN codes [33], respectively (see Fig. S5, Supplemental Material, for more details [4]).

Conclusions. We revisited, theoretically, the point defects in $\mathrm{ZnSnN}_{2}$ by realistic modeling of its disordered phase with the local charge neutrality. Our calculations reveal that the ordered and DLCN models exhibit nearly the same stability, volumes, electronic structures, and even point-defect properties, indicating these properties are determined mainly by the immediate coordination environment. It is also found that low-energy defects with deep levels are absent in $\mathrm{ZnSnN}_{2}$, and therefore there is less carrier recombination caused by point defects than thought previously. Furthermore, a possible route to $\mathrm{ZnSnN}_{2}$ with lower carrier electron concentration using nonequilibrium growth techniques is proposed. Our study unveils the further potential of $\mathrm{ZnSnN}_{2}$ as a photoabsorber in thin-film photovoltaics.

\section{ACKNOWLEDGMENTS}

This work was supported by the MEXT Elements Strategy Initiative to Form Core Research Center, Grants-inAid for Young Scientists A (Grant No. 15H05541) and Scientific Research A (Grant No. 17H01318) from JSPS, and PRESTO (JPMJPR16N4), and Support Program for Starting Up Innovation Hub $\mathrm{MI}^{2}$ I from JST, Japan. The computing resources of ACCMS at Kyoto University were used for a part of this work.

[1] L. Lahourcade, N. C. Coronel, K. T. Delaney, S. K. Shukla, N. A. Spaldin, and H. A. Atwater, Structural and optoelectronic characterization of RF sputtered $\mathrm{ZnSnN}_{2}$, Adv. Mater. 25, 2562 (2013).

[2] S. Lany, A. N. Fioretti, P. P. Zawadzki, L. T. Schelhas, E. S. Toberer, A. Zakutayev, and A. C. Tamboli, Monte carlo simulations of disorder in $\mathrm{ZnSnN}_{2}$ and the effects on the electronic structure, Phys. Rev. Mater. 1, 035401 (2017).

[3] W. Shockley and H. J. Queisser, Detailed balance limit of efficiency of p-n junction solar cells, J. Appl. Phys. 32, 510 (1961).

[4] See Supplemental Material at http://link.aps.org/supplemental/10.1103/PhysRevApplied.10.011001 for details of bulk and defect properties in $\mathrm{ZnSnN}_{2}$.

[5] Y. Hinuma, T. Hatakeyama, Y. Kumagai, L. A. Burton, H. Sato, Y. Muraba, S. Iimura, H. Hiramatsu, I. Tanaka, H. Hosono, and F. Oba, Discovery of earth-abundant nitride semiconductors by computational screening and high-pressure synthesis, Nat. Commun. 7, 11962 (2016).

[6] J. Wu, W. Walukiewicz, S. X. Li, R. Armitage, J. C. Ho, E. R. Weber, E. E. Haller, H. Lu, W. J. Schaff, A. Barcz, and R. Jakiela, Effects of electron concentration on the optical absorption edge of InN, Appl. Phys. Lett. 84, 2805 (2004).

[7] Y. Kumagai, K. Harada, H. Akamatsu, K. Matsuzaki, and F. Oba, Carrier-Induced Band-Gap Variation and Point Defects in $Z n_{3} N_{2}$ from First Principles, Phys. Rev. Appl. 8, 014015 (2017).

[8] Y. Kumagai, N. Tsunoda, and F. Oba, Point Defects and $p$-type Doping in $\mathrm{ScN}$ from First Principles, Phys. Rev. Appl. 9, 034019 (2018).

[9] N. Feldberg, J. D. Aldous, W. M. Linhart, L. J. Phillips, K. Durose, P. A. Stampe, R. J. Kennedy, D. O. Scanlon, G. Vardar, R. L. F. III, T. Y. Jen, R. S. Goldman, T. D. Veal, and S. M. Durbin, Growth, disorder, and physical properties of $\mathrm{ZnSnN}_{2}$, Appl. Phys. Lett. 103, 042109 (2013).

[10] T. D. Veal, N. Feldberg, N. F. Quackenbush, W. M. Linhart, D. O. Scanlon, L. F. J. Piper, and S. M. Durbin, Band gap dependence on cation disorder in $\mathrm{ZnSnN}_{2}$ solar absorber, Adv. Energy Mater. 5, 1501462 (2015).

[11] E. Burstein, Anomalous Optical Absorption Limit in InSb, Phys. Rev. 93, 632 (1954).

[12] T. S. Moss, The interpretation of the properties of indium antimonide, Proc. Phys. Soc. B 67, 775 (1954).

[13] S. Chen, P. Narang, H. A. Atwater, and L.-W. Wang, Phase stability and defect physics of a ternary $\mathrm{ZnSnN}_{2}$ semiconductor: First principles insights, Adv. Mater. 26, 311 (2014).

[14] A. N. Fioretti, A. Zakutayev, H. Moutinho, C. Melamed, J. D. Perkins, A. G. Norman, M. Al-Jassim, E. S. Toberer, and A. C. Tamboli, Combinatorial insights into doping control and transport properties of zinc tin nitride, J. Mater. Chem. C 3, 11017 (2015).

[15] P. C. Quayle, E. W. Blanton, A. Punya, G. T. Junno, K. He, L. Han, H. Zhao, J. Shan, W. R. L. Lambrecht, and K. Kash, Charge-neutral disorder and polytypes in 
heterovalent wurtzite-based ternary semiconductors: The importance of the octet rule, Phys. Rev. B 91, 205207 (2015).

[16] A. N. Fioretti, A. Stokes, M. R. Young, B. Gorman, E. S. Toberer, A. C. Tamboli, and A. Zakutayev, Effects of hydrogen on acceptor activation in ternary nitride semiconductors, Adv. Electron. Mater. 3, 1600544 (2017).

[17] A. Seko and I. Tanaka, Special quasirandom structure in heterovalent ionic systems, Phys. Rev. B 91, 024106 (2015).

[18] J. P. Perdew, A. Ruzsinszky, G. I. Csonka, O. A. Vydrov, G. E. Scuseria, L. A. Constantin, X. Zhou, and K. Burke, Restoring the Density-Gradient Expansion for Exchange in Solids and Surfaces, Phys. Rev. Lett. 100, 136406 (2008).

[19] Y. Kumagai and F. Oba, Electrostatics-based finite-size corrections for first-principles point defect calculations, Phys. Rev. B 89, 195205 (2014).

[20] C. Freysoldt, J. Neugebauer, and C. G. Van de Walle, Fully Ab Initio Finite-Size Corrections for Charged-defect Supercell Calculations, Phys. Rev. Lett. 102, 016402 (2009).

[21] G. Zhang, A. Canning, N. Grnbech-Jensen, S. Derenzo, and L.-W. Wang, Shallow Impurity Level Calculations in Semiconductors using Ab Initio Methods, Phys. Rev. Lett. 110, 166404 (2013).

[22] Y. Kumagai, M. Choi, Y. Nose, and F. Oba, First-principles study of point defects in chalcopyrite $\mathrm{ZnSnP}_{2}$, Phys. Rev. B 90, 125202 (2014).

[23] D. O. Scanlon and A. Walsh, Bandgap engineering of $\mathrm{ZnSnP}_{2}$ for high-efficiency solar cells, Appl. Phys. Lett. 100, 251911 (2012).

[24] D. Skachkov, A. Punya Jaroenjittichai, L.-y. Huang, and W. R. L. Lambrecht, Native point defects and doping in $\mathrm{ZnGeN}_{2}$, Phys. Rev. B 93, 155202 (2016).
[25] J. Neugebauer and C. G. Van de Walle, Hydrogenin GaN: Novel Aspects of a Common Impurity, Phys. Rev. Lett. 75, 4452 (1995).

[26] W. Sun, A. Holder, B. OrvanİLanos, E. Arca, A. Zakutayev, S. Lany, and G. Ceder, Thermodynamic routes to novel metastable nitrogen-rich nitrides, Chem. Mater. 29, 6936 (2017).

[27] Note that Wang et al. previously investigated Li-, Na-, and $\mathrm{K}$-on-Zn substitution as acceptors for $\mathrm{ZnSnN}_{2}$, and concluded that Li-on-Zn substitution acts as a shallow acceptor [34]. However, their study did not calculate the interstitial dopants which act as donors unlike our study.

[28] P. E. Blöchl, Projector augmented-wave method, Phys.Rev. B 50, 17953 (1994).

[29] G. Kresse and D. Joubert, From ultrasoft pseudopotentials to the projector augmented-wave method, Phys. Rev. B 59, 1758 (1999).

[30] G. Kresse and J. Furthmüller, Efficient iterative schemes for ab initio total-energy calculations using a plane-wave basis set, Phys. Rev. B 54, 11169 (1996).

[31] J. Heyd, G. E. Scuseria, and M. Ernzerhof, Erratum: Hybrid functionals based on a screened coulomb potential, J. Chem. Phys. 124, 219906 (2006).

[32] A. V. Krukau, O. A. Vydrov, A. F. Izmaylov, and G. E. Scuseria, Influence of the exchange screening parameter on the performance of screened hybrid functionals, J. Chem. Phys. 125, 224106 (2006).

[33] A. Seko, Y. Koyama, and I. Tanaka, Cluster expansion method for multicomponent systems based on optimal selection of structures for density-functional theory calculations, Phys. Rev. B 80, 165122 (2009).

[34] T. Wang, C. Ni, and A. Janotti, Band alignment and p-type doping of $\mathrm{ZnSnN}_{2}$, Phys. Rev. B 95, 205205 (2017). 